\title{
INVESTIGATION OF INTERCHROMOSOMAL INTERACTIONS AMONG THREE MAJOR CHROMOSOMES OF DROSOPHILA MELANOGASTER IN RESPONSE TO ENVIRONMENTS AND THE RELATIONSHIP BETWEEN MULTI-LINE AND TWO-LINE ANALYSES: RE-EXAMINATION OF CALIGARI AND MATHER DATA
}

\author{
C. S. LIN, C. J. WILLIAMS AND M. R. BINNS
}

Engineering and Statistical Research Institute, Agriculture Canada, Ottawa, K1A OC6

Received 27.ix.83

\section{SUMMARY}

Multi-factor interations in Caligari and Mather's (1975) ANOVA for eight substitution lines of Drosophila melanogaster in nine invironments were examined by cluster analysis. The results indicate a concise explanation of both types of interchromosomal interactions-that which determines the average effect of genotype and that which determines the ordinary genotype-environment (GE) interaction-as follows. For chaeta number, when only one of the three major chromosomes (X, II, and III) of $W$-stock is substituted by $S$-stock, the phenotypic values do not change. However, if more than one chromosome is substituted, they change and differ among themselves. The same pattern holds for yield of offspring with respect to $S$-stock. The data were also investigated by a two-line analysis using an extension of Mather and Caligari's (1976) approach. The statistics for testing the hypothesis of no dependency of GE interaction between a pair of contrasting chromosome (i.e., $g$ values) against the background chromosome, which the pair has in common, are closely related to a component of ANOVA in the eight-line analysis. For those data the $g$ values are shown not to be independent of the background chromosomes. An implication of these analyses is that genes which control average effect and those which control sensitivity to environmental change can be conditionally dependent on other genes of the same type within a chromosome.

\section{INTRODUCTION}

An algebraic formulation of genotype-environment (GE) interaction based on Mendelian concepts was first presented by Mather and Jones (1958). This approach has been expanded greatly in recent years and several hypotheses relating to the genetic structure of GE interaction have been postulated by Professor Mather and his colleagues in Birmingham (e.g., Mather, 1975). One of the key experiments contributing to these developments is Caligari and Mather's (1975) (subsequently referred to as CM75) experiment with Drosophila melanogaster. Eight chromosome substitution lines grown under nine environmental conditions arising from all combinations of three temperatures and three container types were studied for number of sternopleural chaetae and yield of offspring. An important feature of this experiment is that the eight substitution lines were constructed from all possible true-breeding combinations of the three major chromosomes 
$\mathrm{X}, \mathrm{II}$ and III, derived from Wellington (W) and Samarkand (S) inbred stock (i.e., $2^{3}$ factorial combinations).

Since each chromosome was manipulated as a unit in constructing these lines, the data provide a unique opportunity to assess the GE interaction in terms of a polygenic model. In the first (CM75) of two papers, the data were analysed as $2^{3} \times 3^{2}$ factorial experiment. The effect of major chromosomes and their interaction with each other and with environment were examined in terms of Mather and Jinks (1971) genetic parameters. In the second paper, (Mather and Caligari (1976), (MC76)) they analysed the data using Bucio Alanis (1966) two-line analyses, and three statistics were investigated with respect to four sets of different background chromosomes.

The main interest in CM75 and MC76 appears to have been to use this set of data to develop a theory of two types of genes, one controlling "overall effect" (i.e., genetic or average effect), and one controlling "sensitivity to environmental changes" (i.e., GE interaction), and to substantiate the genetical model of $G E$ interaction suggested by Mather (1975). In their analyses, CM75 based their inference almost entirely on the main chromosome effects (a term represented by two genetic parameters $d$ and g, see table 1) for three apparent reasons:

(a) the random error in the ANOVA was deemed to be inappropriate for testing genetic parameters derived from comparison among genotypes, (in CM75's notation, these include all $d$ 's and $d \times d s$, see table 1). (When a more appropriate error was used the number of significant interactions was reduced).

(b) the mean squares (MS) for main chromosomal effects were generally much larger than for the interchromosomal interaction effects.

(c) the two-line analyses suggested that the $G E$ interaction between pairs of contrasting chromosomes were unaffected by the background chromosomes in all instances except one.

Although the average differences between genotypes are clearly very important aspects in the interpretation of these data, it is also true that some of the two-factor interactions are significant even when the larger error variance estimated from the related diallel experiment was used; and several three-factor interactions are significantly larger than the random error (which CM75 considered appropriate for these effects). It is therefore worth considering the possible difference in interpretation of the whole experiment if the relatively large interactions are considered to be real.

Interpretation is, however, complicated because significant interactions are linked by common factors: in a factorial experiment, if the factors involved in significant interactions are mutually exclusive (e.g., $A \times B, C \times D$ in a $A \times B \times C \times D$ factorial design) each interaction can be interpreted separately, but, if they are interrelated by a common factor (e.g., $A \times B$, $B \times C)$, separate investigations of each interaction may not reveal the relationship among factors since factor $A$ depends on $B$, and $B$ depends on $C$. Under these circumstances the overall interaction structure should be investigated for all factors involved in the interactions. This means that for the present data at least four factor interactions need to be investigated. However, the examination of these relationships cannot be done easily by using tables of means. The strategy we adopt is to re-define the design as a two-way classification of two super factors ( 8 genotypes in 9 environments) 
TABLE 1

ANOVA's for $2^{3} \times 3^{2}$ factorial experiment based on table 2

\begin{tabular}{|c|c|c|c|c|}
\hline \multirow[b]{2}{*}{ Source } & \multirow[b]{2}{*}{$\begin{array}{l}\text { CM75's } \\
\text { notation }\end{array}$} & \multirow[b]{2}{*}{ df } & \multicolumn{2}{|c|}{ MS } \\
\hline & & & $\begin{array}{l}\text { Chaeta } \\
\text { number }\end{array}$ & $\begin{array}{l}\text { Yield of } \\
\text { offspring }\end{array}$ \\
\hline Genetic effects $(G)$ & & 7 & & \\
\hline $\mathbf{X}$ & $d_{\mathbf{x}}$ & 1 & $5.2434\left({ }^{* *}\right) \dagger$ & 453 \\
\hline II & $\widehat{d_{1 I}}$ & 1 & 1.4993 & $89782^{* *}$ \\
\hline III & $d_{1 I I}$ & 1 & $43.4156(* *)$ & $13028^{* *}$ \\
\hline $\mathrm{X} \times \mathrm{II}$ & $d_{\mathbf{x}} \times d_{\mathrm{II}}$ & 1 & $8.5905\left(^{* *}\right)$ & $21064^{* *}$ \\
\hline $\mathrm{X} \times \mathrm{III}$ & $d_{\mathbf{x}} \times d_{1 \mathrm{II}}$ & 1 & $20 \cdot 5120\left(^{* *}\right)$ & 3620 \\
\hline II $\times$ III & $d_{I I} \times d_{I I I}$ & 1 & $0 \cdot 0101$ & $13434^{* *}$ \\
\hline $\mathrm{X} \times \mathrm{II} \times \mathrm{III}$ & $d_{\mathbf{X}} \times d_{\mathrm{II}} \times d_{\mathrm{III}}$ & 1 & $0 \cdot 0058$ & 803 \\
\hline Environmental effects & & 8 & & \\
\hline Temperatures $(T)$ & & 2 & $8 \cdot 5596^{* *}$ & $18582^{* *}$ \\
\hline Containers $(C)$ & & 2 & $2 \cdot 4738^{* *}$ & $138550^{* *}$ \\
\hline$T \times C$ & & 4 & $0 \cdot 2325$ & 4885 \\
\hline$G \times E$ interaction & & 56 & & \\
\hline $\mathrm{X} \times T$ & $g_{d x}$ & 2 & $0.4705^{*}$ & 365 \\
\hline $\mathrm{II} \times T$ & $\mathbf{g}_{d I I}$ & 2 & $4 \cdot 1047^{* *}$ & 1571 \\
\hline III $\times T$ & $g_{d \text { III }}$ & 2 & $0.7272 *$ & 1295 \\
\hline $\mathrm{x} \times C$ & $g_{d x}$ & 2 & 0.0586 & 155 \\
\hline II $\times C$ & $\begin{array}{l}\boldsymbol{g}_{d \mathbf{}} \\
\boldsymbol{g}_{d \mathbf{x}}\end{array}$ & 2 & $0 \cdot 1765$ & $24973^{* * *}$ \\
\hline III $\times C$ & $g_{d 111}$ & 2 & 0.0470 & $11846^{* *}$ \\
\hline $\mathrm{X} \times \mathrm{II} \times T$ & $g_{d x \times d I I}$ & 2 & 0.0230 & 5267 \\
\hline $\mathrm{X} \times \mathrm{III} \times T$ & $g_{d x \times d I I I}$ & 2 & $0.3062^{*}$ & 3782 \\
\hline $\mathrm{II} \times \mathrm{III} \times T$ & $\boldsymbol{g}_{d \mathrm{H} \times d \mathrm{HI}}$ & 2 & $0 \cdot 3480^{*}$ & 3080 \\
\hline $\mathrm{X} \times \mathrm{II} \times C$ & $g_{d x \times d 11}$ & 2 & $0 \cdot 1061$ & $8252^{*}$ \\
\hline $\mathrm{X} \times \mathrm{III} \times C$ & $g_{d x \times d I I I}$ & 2 & 0.0020 & 1983 \\
\hline $\mathrm{II} \times \mathrm{III} \times C$ & $g_{d I 1 \times d 11 I}$ & 2 & 0.1214 & $7794^{*}$ \\
\hline $\mathrm{X} \times T \times C$ & & 4 & $0 \cdot 1093$ & 1677 \\
\hline $\mathrm{II} \times T \times C$ & & 4 & 0.0131 & 1768 \\
\hline III $\times T \times C$ & & 4 & 0.0279 & 941 \\
\hline Pooled 4-fact Int. & & 16 & 0.0793 & 1825 \\
\hline $\mathrm{X} \times \mathrm{II} \times \mathrm{III} \times T \times C$ & & 4 & 0.0439 & 2448 \\
\hline Errorł & & 72 & 0.0959 & 2246 \\
\hline
\end{tabular}

* Significant at 5 per cent.

** Significant at 1 per cent.

$\dagger$ Parenthesis indicate test results based on the error estimates from diallel cross $(1 \cdot 3943 / 4=$ $0.3486)$.

$\ddagger$ Derived from the figures given by Caligari and Mather (1975): $0 \cdot 3855 / 4=0.0957$, and $4491 / 2=2246$ respectively.

and group the genotypes based on their similarity of average effect and sensitivity to environmental change. The interaction structure of the original factors is then examined through the pattern of the factorial configuration within each group. This approach was found to be successful for this set of data and a systematic pattern of the interaction structure was identified.

The purposes of this paper are to demonstrate the logical development of the analyses which led to this identification and to present a reformulation of the two-line analysis relating to the eight-line analysis showing how, in certain circumstances, a real interaction effect can remain undetected. The role of clustering in exploratory data analysis and an alternative interpretation in terms of the theory of two types of genes based on the present finding are discussed. 


\section{DAta AND Cluster ANAlyses}

\section{(i) Experimental data}

The mean chaeta number and mean yield of offspring for eight genotypes in nine environments are shown in table 2 (table 5 of CM75). Each genotype is represented by three letters indicating the origin ( $W$ or $S$ ) of the chromosomes X, II and III in that order. For example, WSS represents the genotype with its $\mathrm{X}$-chromosome from $W$-stock and chromosomes II and III from $S$-stock. The ANOVA's for the $2^{3} \times 3^{2}$ factorial (table 1) show that the factors involved in significant interactions are interrelated. Therefore, four-factor interactions (three major chromosomes and one environmental factor) need to be investigated for each trait.

TABLE 2

Mean chaeta numbers and yields of offspring of eight genotypes in nine environments, table 5 in Caligari and Mather (1975)

\begin{tabular}{|c|c|c|c|c|c|c|c|c|c|}
\hline \multirow{2}{*}{$\frac{\text { Container types }}{\text { Temperatures }}$} & \multicolumn{3}{|c|}{ B } & \multicolumn{3}{|c|}{$Y$} & \multicolumn{3}{|c|}{$\mathbf{U}$} \\
\hline & $25^{\circ} \mathrm{C}$ & $21 \cdot 5^{\circ} \mathrm{C}$ & $18^{\circ} \mathrm{C}$ & $25^{\circ} \mathrm{C}$ & $21 \cdot 5^{\circ} \mathrm{C}$ & $18^{\circ} \mathrm{C}$ & $25^{\circ} \mathrm{C}$ & $21.5^{\circ} \mathrm{C}$ & $18^{\circ} \mathrm{C}$ \\
\hline Genotypes & \multicolumn{9}{|c|}{ Chaeta numbers } \\
\hline SSS & $20 \cdot 30$ & $20 \cdot 23$ & $19 \cdot 90$ & $20 \cdot 15$ & $19 \cdot 42$ & 19.85 & 19.97 & $19 \cdot 50$ & $19 \cdot 55$ \\
\hline WSS & $19 \cdot 20$ & $19 \cdot 52$ & 18.95 & $19 \cdot 15$ & $19 \cdot 05$ & $18 \cdot 88$ & $18 \cdot 33$ & $18 \cdot 50$ & $18 \cdot 88$ \\
\hline SWS & 20.08 & $21 \cdot 37$ & $21 \cdot 25$ & $20 \cdot 48$ & $21 \cdot 00$ & 21.95 & 19.75 & $20 \cdot 33$ & $21 \cdot 10$ \\
\hline$S S W$ & 16.85 & 18.48 & $17 \cdot 37$ & 16 & $17 \cdot 15$ & 17 & $16 \cdot 38$ & $17 \cdot 40$ & 16.95 \\
\hline$S W W$ & $17 \cdot 27$ & $19 \cdot 10$ & 18.85 & $17 \cdot 30$ & $18 \cdot 65$ & $19 \cdot 20$ & $16 \cdot 60$ & 18.20 & 18.93 \\
\hline WSW & $18 \cdot 18$ & $19 \cdot 25$ & 19. & 17.67 & $18 \cdot 58$ & $19 \cdot 12$ & 17.57 & 18.05 & 18.05 \\
\hline$W W S$ & $17 \cdot 50$ & $19 \cdot 22$ & $19 \cdot 80$ & $17 \cdot 32$ & $18 \cdot 80$ & 19.92 & 16.53 & $18 \cdot 75$ & 18.95 \\
\hline$W W W$ & $16 \cdot 80$ & 18.50 & $19 \cdot 40$ & $17 \cdot 10$ & $18 \cdot 28$ & $18 \cdot 80$ & $16 \cdot 68$ & $18 \cdot 28$ & 18.63 \\
\hline $\begin{array}{l}\text { Environment } \\
\text { indices }\end{array}$ & -0.49 & $0 \cdot 70$ & 0.62 & $0 \cdot 50$ & $0 \cdot 10$ & 0.63 & -1.04 & $-0 \cdot 14$ & .12 \\
\hline Genot) & \multicolumn{9}{|c|}{ Yield of offspring } \\
\hline SSS & $190 \cdot 0$ & $153 \cdot 5$ & $47 \cdot 5$ & $131 \cdot 5^{\circ}$ & 150.5 & 37.5 & $25 \cdot 5$ & $29 \cdot 0$ & $11 \cdot 5$ \\
\hline & $117 \cdot 0$ & $127 \cdot 5$ & 94.5 & $75 \cdot 0$ & $91 \cdot 0$ & $70 \cdot 0$ & $71 \cdot 0$ & $31 \cdot 5$ & $24 \cdot 0$ \\
\hline$s V$ & $130 \cdot 5$ & $119 \cdot 0$ & $180 \cdot 5$ & $127 \cdot 5$ & $97 \cdot 5$ & $106 \cdot 0$ & $66 \cdot 5$ & $43 \cdot 0$ & $48 \cdot 5$ \\
\hline$S S W$ & $198 \cdot 5$ & 253.0 & $111 \cdot 5$ & $47 \cdot 5$ & $84 \cdot 0$ & $105 \cdot 0$ & $19 \cdot 5$ & $111 \cdot 0$ & $30 \cdot 5$ \\
\hline$S W W$ & $391 \cdot 5$ & $385 \cdot 5$ & $153 \cdot 5$ & $116 \cdot 0$ & $183 \cdot 0$ & $80 \cdot 0$ & $55 \cdot 0$ & 63.0 & $46 \cdot 5$ \\
\hline WSW & $80 \cdot 0$ & $57 \cdot 0$ & $89 \cdot 5$ & $56 \cdot 0$ & $63 \cdot 0$ & $65 \cdot 5$ & $29 \cdot 0$ & 34.5 & $35 \cdot 5$ \\
\hline WWS & 276.5 & $356 \cdot 0$ & 121 & $119 \cdot 0$ & $194 \cdot 5$ & $107 \cdot 5$ & $84 \cdot 5$ & $49 \cdot 0$ & $30 \cdot 5$ \\
\hline$W W W$ & $327 \cdot 5$ & $495 \cdot 0$ & $300 \cdot 5$ & $136 \cdot 5$ & $161 \cdot 5$ & $138 \cdot 0$ & $105 \cdot 0$ & $52 \cdot 0$ & $43 \cdot 5$ \\
\hline indices & $96 \cdot 7$ & $126 \cdot 1$ & $20 \cdot 2$ & $-16 \cdot 1$ & $10 \cdot 9$ & $-28 \cdot 5$ & $-60 \cdot 2$ & $-65 \cdot 6$ & $-83 \cdot 4$ \\
\hline
\end{tabular}

\section{(ii) Clustering procedure}

To investigate the overall interaction structure, the design was defined as a two-way classification of eight genotypes $(2 \times 2 \times 2)$ by nine environments $(3 \times 3)$. Each genotype (substitution line) was regressed on an environmental index, calculated as the difference between the mean response at a particular environment and the overall mean (table 2), and clusters were obtained by the method of Lin and Thompson (1975). 
Five groups for chaeta number and four groups for yield of offspring were formed when the grouping was stopped after the dissimilarity index (defined as the sum of squares of deviations from common regression) exceeded the tabulated 5 per cent $F$-value. The results of the clustering process are shown by the dendrogram in fig. 1 .

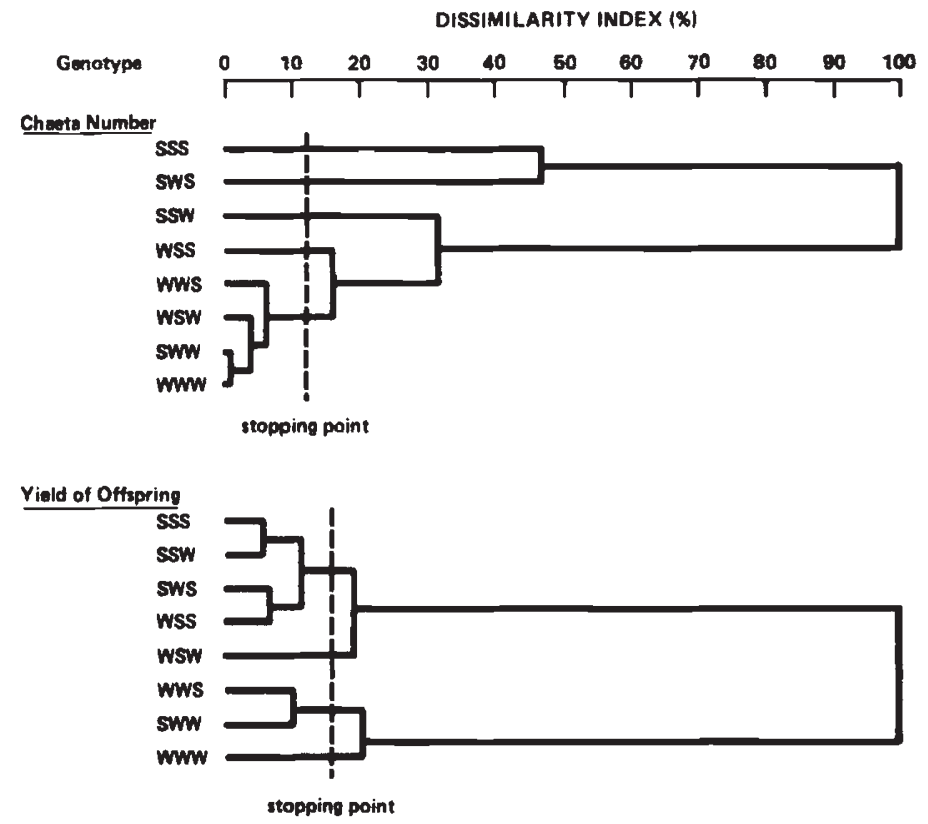

FIG. 1. Dendrogram of clustering process for eight genotypes based on Lin and Thompson's method.

(iii) Cluster pattern and the structure of the data

The general pattern underlying the chromosome constitution of these groups is as follows:

(a) With the exception of $S W W$ and $W W S$ for yield of offspring, the groups for chaeta number and for yield of offspring are complementary in the sense that genotypes belonging to the same group for one trait are in different groups for the other trait. This result suggests that these two traits are independent (the correlation coefficient between the traits is only -0.04 from the observed values and -0.05 from residuals after fitting the $2^{3} \times 3^{2}$ factorial model).

(b) For chaeta number, all genotypes possessing at least two chromosomes from the $W$-stock responded to environment similarly (i.e., $W W W=S W W=W S W=W W S$ ). We can express this concisely by saying that $W$-stock prevails over $S$-stock for chaeta number. By contrast, for yield of offspring, all genotypes possessing at least two chromosomes from the $S$-stock responded to environment similarly; (i.e., $S S S=W S S=S W S=S S W$ ). Thus the $S$-stock prevails over the $W$-stock for yield of offspring. 
(c) For chaeta number, all genotypes possessing at most one chromosome from $W$-stock responded to environment differently; (i.e., $S S S \neq W S S \neq S W S \neq S S W$ ). From yield of offspring, all genotypes possessing at most one chromosome from $S$-stock responded to environment in one of three ways; (i.e., $W W W \neq S W W=W W S \neq$ WSW).

\section{(iv) Examination of two sets of genotypes}

The groupings were obtained by a method which assumes a linear regression of $\mathrm{GE}$ interaction on the environmental index. To check whether the conjectured data structure is consistent with the original environmental factors, i.e., container and temperature, the data were analysed with the genotypes divided into two sets, the first consisting of genotypes with at most one $W(S S S, W S S S W S, S S W)$ and the second consisting of genotypes with at most one $S(W W W, S W W, W S W, W W S)$. The analyses (table 3$)$

TABLE 3

ANOVAS based on the factorial model of Genotype $\times$ Container $\times$ Temperature, Genotype set $1\left(G_{1}\right)$ : SSS, WSS, SWS, SSW; Genotype set $2\left(G_{2}\right): W W W, S W W$, WSW, WWS

\begin{tabular}{|c|c|c|c|}
\hline \multirow[b]{2}{*}{ Source } & \multirow[b]{2}{*}{ df } & \multicolumn{2}{|c|}{ MS } \\
\hline & & Chaeta number & $\begin{array}{l}\text { Yield of } \\
\text { offspring }\end{array}$ \\
\hline Genotypes $(G)$ & 7 & $11 \cdot 3252$ & 20312 \\
\hline$G_{1}$ & 3 & $21 \cdot 2179$ & 1626 \\
\hline$G_{2}$ & 3 & 0.4225 & 31985 \\
\hline$G_{1}$ vs. $G_{2}$ & 1 & $14 \cdot 3558$ & 41352 \\
\hline Containers $(C)$ & 2 & $2 \cdot 4738$ & 138550 \\
\hline Temperatures $(T)$ & 2 & $8 \cdot 5596$ & 18582 \\
\hline$C \times T$ & 4 & 0.2325 & 4885 \\
\hline$G \times C$ & 14 & 0.0795 & 7951 \\
\hline$G_{1} \times C$ & 6 & 0.0838 & 1482 \\
\hline$G_{2} \times C$ & 6 & 0.0953 & 11308 \\
\hline$\left(G_{1}\right.$ vs. $\left.G_{2}\right) \times C$ & 2 & 0.0193 & 17285 \\
\hline$G \times T$ & 14 & 0.8730 & 2738 \\
\hline$G_{1} \times T$ & 6 & 0.5552 & 2553 \\
\hline$G_{2} \times T$ & 6 & 0.2651 & 3105 \\
\hline$\left(G_{1}\right.$ vs. $\left.G_{2}\right) \times T$ & 2 & $3 \cdot 6496$ & 2290 \\
\hline$G \times C \times T$ & 28 & 0.0605 & 1700 \\
\hline$G_{1} \times C \times T$ & 12 & 0.0340 & 1244 \\
\hline$G_{2} \times C \times T$ & 12 & 0.0764 & 2052 \\
\hline$\left(G_{1}\right.$ vs. $\left.G_{2}\right) \times C \times T$ & 4 & 0.0921 & 2015 \\
\hline Replicate error $\dagger$ & 72 & 0.0959 & 2246 \\
\hline
\end{tabular}

$\dagger$ Derived from the figures given by Caligari and Mather (1975);0.3835/4 = 0.0959 , and $4491 / 2=2246$ respectively.

show that most of the variation among genotypes and most of the variation associated with their interaction with environment (temperature for chaeta number and container type for yield of offspring) is accounted for by set 1 for chaeta number, by set 2 for yield of offspring, and by the contrast 
between sets for both traits. The variation accounted for by the complementary sets and the corresponding sources is generally small, i.e., about the same magnitude as the replicate error.

The appropriateness of the postulated two set structure can also be conveniently shown by plotting the data, separately for each set, in the following way: chaeta number, averaged over container type, against temperature (fig. 2) and yield of offspring averaged over temperature, against container type (fig. 3). The similarity of responses of the four genotypes in set 2 for chaeta number and in set 1 for yield of offspring is clearly indicated. Hence it seems reasonable to conclude that the interchromosomal interaction arises because the phenotypic value of the prevailing stock remains unchanged if only one chromosome is substituted.

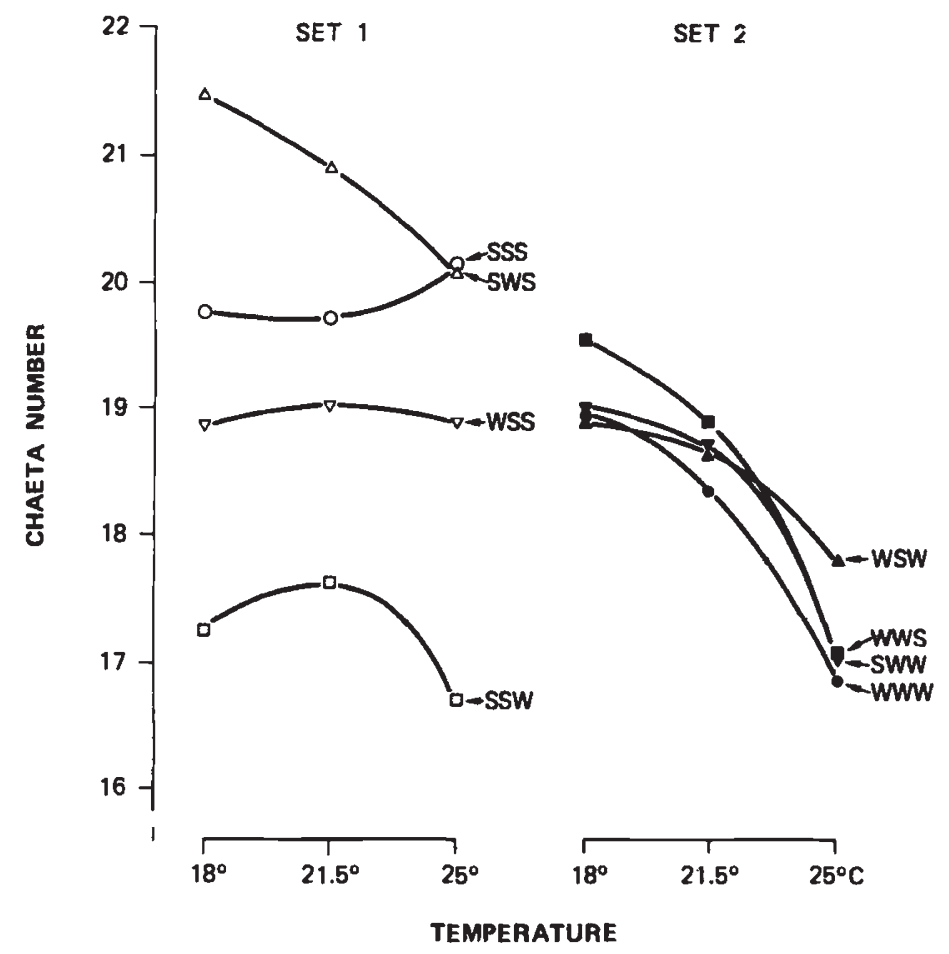

FIG. 2. Mean chaeta number for each temperature level, averaged over container type.

\section{INVESTIGATION OF GE INTERACTION UNDER FOUR SETS OF BACKGROUND CHROMOSOME BASED ON TWO-LINE MODEL}

Bucio Alanis' (1966) two-line model includes three parameters: an overall effect $(d)$ which is the difference between two genotypes averaged over environments, an environmental effect $(e)$ which is the difference between environments averaged over genotypes, and the statistical interaction $(g$ ) between $d$ and $e$ (i.e., GE interaction). MC76 studied the characteristics of these parameters in the following way. For each of the three pairs of contrasting chromosomes ( $W$ vs. $S$ for X, II, III), taken in turn, 


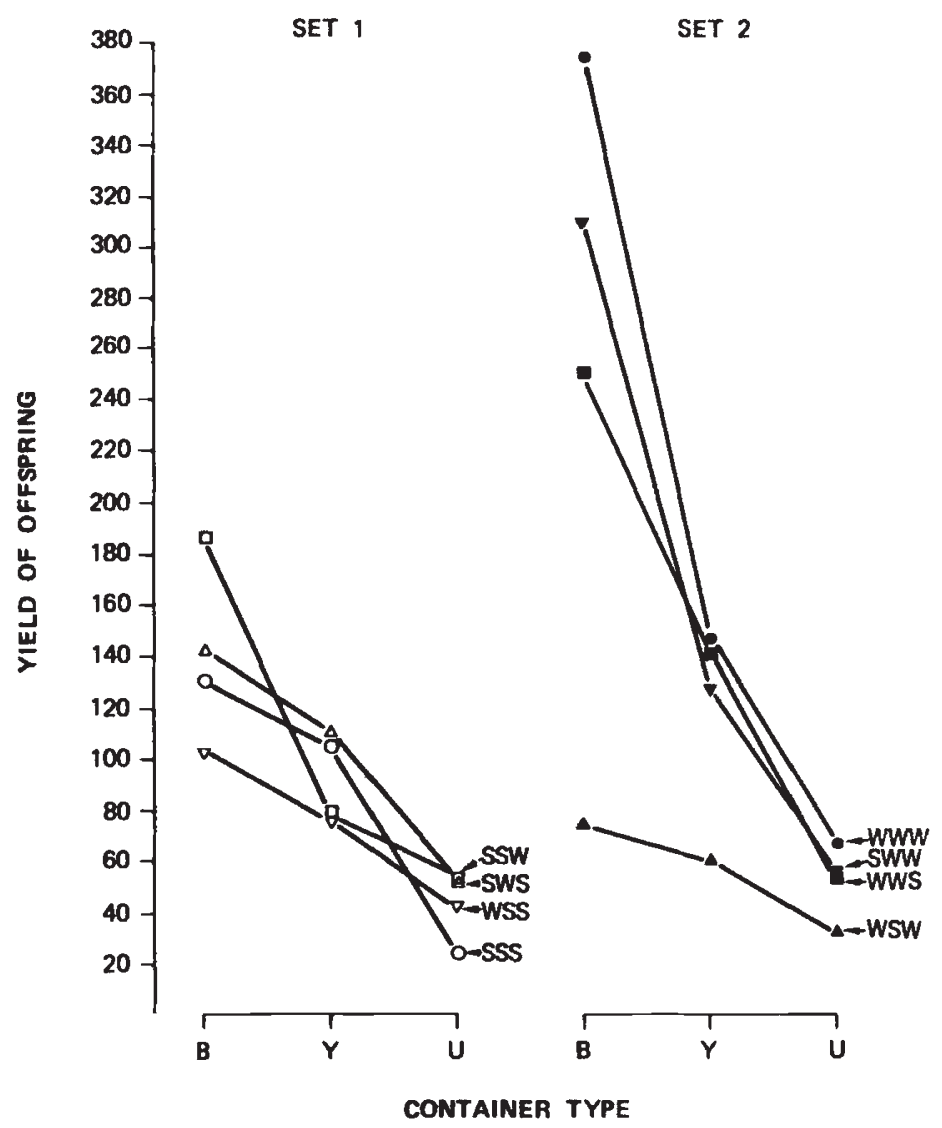

FIG. 3. Mean yield of offspring for each container type, averaged over temperature.

the eight substitution lines were divided into four sets corresponding to all possible combinations of the other two chromosomes, so that two genotypes in the same set were alike with respect to these background chromosomes (e.g., for X-chromosome, the eight lines yield four sets: $W W W$ vs. $S W W$, $W \underline{W S}$ vs. $\underline{S W S}, W \underline{S W}$ vs. $S \underline{S W}, W \underline{S S}$ vs $\underline{S S}$, where background chromosomes are underlined).

To study whether $g$ values are influenced by the background chromosomes, the two-line model can be defined in the following way. Let $d_{\mathrm{x}}, e_{\mathrm{x}}, g_{\mathrm{x}}$ represent the genetic, environment and GE interaction respectively for the $\mathrm{X}$-chromosome, and let $d_{B i}, e_{B i}, g_{B i}$ represent the corresponding values for background chromosome $B_{i}$, (where $i=1, \ldots, 4$ corresponding to 4 sets of background chromosomes $-W W,-W S,-S W,-S S$ respectively). Further if we write $g_{X} \times g_{B i}$ interaction as $I_{X B i}$, then two genotypes which differ in their X-chromosome but with a uniform background $B_{i}$ in two environments can be written as in table 4 .

Table 4 is basically similar to table 4 of MC76 except that the parameters $I_{X B}$ are now included. For setting whether $g$ values are interacting with background chromosomes, MC76 compared the sum of squares $(S S)$ of differences among four sets of backgrounds using Bartlett's test. They felt 
TABLE 4

The parameter structure of two genotypes different at $X$-chromosome at two environments

\begin{tabular}{|c|c|c|c|}
\hline \multirow{2}{*}{$\begin{array}{l}\text { Genotype } \\
(i=2)\end{array}$} & \multicolumn{2}{|c|}{ Environment } & \multirow[b]{2}{*}{ mean } \\
\hline & 1 & 2 & \\
\hline$W W W$ & $d_{\mathrm{X}}-e_{\mathrm{X}}-e_{\mathrm{B} i}-g_{\mathrm{X}}-g_{B}-I_{\mathrm{X} B}$ & $d_{\mathrm{X}}+e_{\mathrm{X}}+e_{B i}+g_{\mathrm{X}}+g_{B}+\mathrm{I}_{\mathrm{X} B i}$ & $d_{\mathbf{X}}$ \\
\hline$S W W$ & $-d_{\mathrm{X}}-e_{\mathrm{X}}-e_{B i}+g_{\mathrm{X}}-g_{B}+\mathrm{I}_{\mathrm{X} B}$ & $-d_{\mathrm{X}}-e_{\mathrm{X}}+e_{B i}-g_{\mathrm{X}}+g_{B}-I_{\mathrm{X} B i}$ & $-d_{\mathrm{x}}$ \\
\hline $\begin{array}{l}\text { Sum } \\
\text { Difference }\end{array}$ & $\begin{array}{c}2\left(-e_{\mathrm{X}}-e_{B i}-g_{B i}\right) \\
2\left(d_{\mathbf{X}}-g_{\mathbf{X}}-\mathrm{I}_{\mathbf{X} B}\right)\end{array}$ & $\begin{array}{l}2\left(+e_{\mathrm{X}}+e_{B i}+g_{B}\right) \\
2\left(d_{\mathrm{X}}+g_{\times} \times \mathrm{I}_{\mathbf{X} B i}\right)\end{array}$ & $\begin{array}{c}0 \\
2 d\end{array}$ \\
\hline
\end{tabular}

$S S$ of Sum $=4\left(e_{\mathrm{X}}+e_{B i}+g_{B i}\right)^{2}$.

$S S$ of Difference $=4\left(g_{X}+I_{X B i}\right)^{2}$.

Note MC76's notations corresponding to the present notations in the following way (MC76's notations are left):

$$
\begin{aligned}
e_{x w} & =e_{x}+g_{x} \\
e_{x s} & =e_{x}-g_{x} \\
e_{B} & =e_{B i}+g_{B}
\end{aligned}
$$

that if no interaction were present (i.e., $I_{\mathrm{X} B}=0$ ) the $S S$ of differences should be approximately homogeneous for four sets.

$$
S S \text { of difference }=4\left(g_{\mathrm{X}}+I_{\mathrm{XBi}}\right)^{2} \text {. }
$$

However as equation (1) shows, the $S S$ of differences contains two components, so the test may not be very sensitive since a relatively large value of $g$ can easily mask the effect of $I_{\mathrm{XB}}$. A more sensitive test is to calculate the $S S$ of $\left(I_{X B i}\right)$ for all four sets as follows:

$$
\sum_{i=1}^{4}\left(S S \text { of } I_{X B i}\right)=\sum_{i=1}^{4}(S S \text { of Diff })_{i}-S S \text { (overall Diff) }
$$

where $\quad S S$ (overall difference) $=S S(W W W+W W S+W S W+W S S-$ $S W W-S W S-S S W-S S S)$.

It is interesting to note that $\sum_{i}\left(S S\right.$ of $\left.I_{X B i}\right)$ can be written in terms of the $S S$ from the ANOVA based on the four-factor factorial experiment $(\mathrm{X} \times \mathrm{II} \times \mathrm{III} \times E)$ in the following way:

$$
\begin{aligned}
\sum_{i=1}^{4}\left(S S \text { of } I_{\mathrm{X} B i}\right)= & S S(\mathrm{X} \times \mathrm{II} \times E)+S S(\mathrm{X} \times \mathrm{III} \times E) \\
& +S S(\mathrm{X} \times \mathrm{II} \times \mathrm{III} \times E) \\
\sum_{i=1}^{4}\left(S S \text { of } I_{\mathrm{IIBi}}\right)= & S S(\mathrm{II} \times \mathrm{X} \times E)+S S(\mathrm{II} \times \mathrm{III} \times E) \\
& +S S(\mathrm{X} \times \mathrm{II} \times \mathrm{III} \times E) \\
\sum_{i=1}^{4}\left(S S \text { of } I_{\mathrm{IIIBi}}\right)= & S S(\mathrm{III} \times \mathrm{X} \times E)+S S(\mathrm{III} \times \mathrm{II} \times E) \\
& +S S(\mathrm{X} \times \mathrm{II} \times \mathrm{III} \times E) .
\end{aligned}
$$

The MS of (3), (4) and (5) can be tested for significance against the replicate error. However, like the tests for homogeneity of the three statistics used by MC76, these tests are not independent. 
Before applying these test statistics to the data, it is important to recall that since only one contrast of one environmental factor appears to be involved in the $\mathrm{GE}$ interaction, i.e., $T_{2}\left(18^{\circ} \mathrm{C}\right.$ and $21.5^{\circ} \mathrm{C}$ vs. $\left.25^{\circ} \mathrm{C}\right)$ for chaeta number and $C_{2}$ ( $Y$ and $U$ vs. $B$ ) for yield of offspring (see tables 6 and 9 of CM75), it seems reasonable to test the $M S$ for $I_{\mathrm{XB}}$ using these two environmental contrasts, as appropriate, for the two traits. However, to demonstrate how the choice of environmental conditions may influence results, we also present the $I_{X B}$ interaction $M S$ using all nine environments $(E)$, as well as using the significant environmental factor, (i.e., temperature $(T)$ for chaeta number and container type $(C)$ for yeild of offspring). The results (table 5) show that when the $M S$ are calculated based on $E$ none are significant, but when based on $T$ or $C$ two are significant. Finally, when based on $T_{2}$ or $C_{2}$ the null hypotheses were rejected, with one exception, for all chromosomes and both traits.

TABLE 5

The MS for $g \times$ background interactions measured under three environmental conditions: $E$, $T($ or $C)$, and $T_{2}\left(\right.$ or $\left.C_{2}\right)$, defined in the text

\begin{tabular}{|c|c|c|c|c|c|c|c|}
\hline \multirow[b]{2}{*}{$\begin{array}{l}\text { Contrasting } \\
\text { chromosome }\end{array}$} & \multirow[b]{2}{*}{ (df) } & \multicolumn{3}{|c|}{ Chaeta Number } & \multicolumn{3}{|c|}{ Yield of Offspring } \\
\hline & & $\begin{array}{c}E \\
(24)\end{array}$ & $\begin{array}{c}T \\
(6)\end{array}$ & $\begin{array}{l}T_{2} \\
\text { (3) }\end{array}$ & $\underset{(24)}{E}$ & $\begin{array}{c}C \\
(6)\end{array}$ & $\begin{array}{l}C_{2} \\
{ }_{(3)}\end{array}$ \\
\hline $\mathrm{x}$ & & 0.085 & $0 \cdot 153$ & 0.202 & 3077 & 3630 & $6478^{*}$ \\
\hline II & & 0.095 & $0 \cdot 167$ & $0 \cdot 296^{*}$ & 3259 & $5567^{*}$ & $10067^{* *}$ \\
\hline III & & $0 \cdot 113$ & $0 \cdot 262^{*}$ & $0.420^{* *}$ & 2718 & 3477 & $6223^{*}$ \\
\hline$M S$ of error & 72 & & 0.0959 & & & 2246 & \\
\hline
\end{tabular}

MC76's conclusion that $g$ was unaffected by backgrounds (except chromosome III for yield of offspring) can be attributed to the test statistics and to the inclusion of all nine environments.

\section{DisCUSSION AND CONCLUSION}

One of the most important objectives in the investigation of significant multi-factor interactions is to find out if there is any systematic pattern with respect to factor configurations. Such evidence, if recognised, can be a valuable scientific finding; if not recognized, the analysis may suggest an incorrect conclusion.

The identification of interaction patterns in the present example is due in large measure to the fact that these multifactors can be neatly classified into two super-factors, genotype and environment; and that the characteristics of the interaction served so well in differentiating the genotypes of different chromosome configurations. When a variable is highly susceptible to environmental impact (or other factor), the use of these responses as the criterion for grouping can be a useful tool for some biological problems. The similarity of response over a range of environments may indicate a common biological constitution. For example, Lin et al., (1977) grouped 12 carp genotypes of known genetic background and found that the grouping 
based on phenotypic values in five environments closely reflected the genetic background.

It is important to emphasise that cluster analysis is only a systematic way of finding regularity or patterns in data. The clusters themselves need not have an intrinsic meaning, but may suggest useful generalisations about the structure of the data; for example, in the present case, the two sets of genotypes. Anderberg's (1973) remark that "once a satisfactory structure is known and defended on its own merits any cluster analysis that contributed to its discovery is only of historical interest" is an excellent statement of this role of cluster analysis. Essentially, cluster analysis is a tool of discovery about the data. This is the basic attitude toward cluster analysis as employed in the present paper.

Another point to be noted is that clustering is an exploratory procedure whose primary function is to propose an explanation from the data; ANOVA is a confirmatory method whose primary function is to test whether the data conform with a proposed explanation: i.e., clustering is hypothesisgenerating and ANOVA is hypothesis-testing. In research, one needs to be aware of the limitations of confirmatory methods as tools for scientific inference. This is particularly true when dealing with new types of data, since, if the underlying mechanisms generating data are not fully understood, there is a possibility of relationships being overlooked if the only methods used in the analyses are those which conform with an existing hypothesis or model.

The present analyses show that the interchromosomal interaction in this set of data has an intrinsic pattern with respect to the chromosome configurations. The interaction apparently arises because the phenotypic values of prevailing (defined above) stocks remain unchanged if only one chromosome is substituted. Such a homeostatic property makes data interpretation based only on main chromosome effects uninformative. The summary for individual chromosome effect given in Table 11 of CM75 may be true for four genotypes with at most one chromosome from the prevailing stock, but untrue for the other four. For example, chromosome III was described as exerting the largest "average effect" on chaeta number. This is true when the effect is measured by the contrast $S S W-S S S$, (difference $=-2 \cdot 66 \pm 0 \cdot 28$, the S.E. being based on the error variance 1.39/4); but untrue when measured by the contrast WSW-WSS (difference $=-0.49$ ). The inconsistency strongly suggests that the interaction is fully as important as the main effect in interpreting the data.

Since the two-line analysis and the ANOVA for all eight genotypes are statistically related, as shown by equations (2) and (3), the conclusions derived from the two methods are expected to be the same. Nevertheless, MC76's investigation using the two-line analysis is genetically useful, because it provides an opportunity for assessing the validity of Mather (1975) and CM75's genetic hypotheses on GE interactions. Although three statistics $(e, b, g)$ were investigated by MC76 over the four sets of background chromosomes, the main issue, from the viewpoint of Mather's (1975) genetic model, is whether the $g$ of the contrasting chromosomes was independent of the background. Our investigation has shown that $g$ was not independent. Thus Mather's (1975) expectation that "genes which do not differ between the genotypes, can affect the biological assessment $(e)$ of the environment; but they obviously cannot produce any genotype $x$ 
environment interaction $(g)$ " is not supported by the data. That is the $g$ for a particular fixed background may be different from the $g$ for some other fixed background. Although not shown here, similar investigations conducted for the genetic parameter $d$ also showed that $d$ was not independent of the background chromosomes.

When interchromosome interactions exist, interpretation based on main effects only may be misleading. If we were to assume that the genes controlling average effects and the genes controlling GE interactions are of two distinct types and that what has happened at the chromosome level can be extended to the gene level (as CM75 conjectured), then the implication from the present data is that both types of genes can be conditionally dependent on the other genes of the same type within a chromosome; under certain gene combinations, the interaction effect among genes (i.e., epistasis) could cancel the individual gene effects.

\section{REFERENCES}

ANDerberg, M. 1973. Cluster Analysis for Application. Academic Press, New York.

BUCIO ALANIS, 1. 1966. Environmental and genotype-environmental components of variability. I. Inbred lines. Heredity, 21, 287-397.

CALIGARI, P. D. S. AND MATHER, K. 1975. Genotype $\times$ environment interactions. III. Interactions in Drosophila melanogaster. Proc. Roy. Soc. B., 191, 387-411.

LIN, C. S., BINNS, M. R. AND THOMPSON, B. K. 1977. The use of regression methods to study genotype-environment interactions-Extending Griffing's model for diallel cross experiment and testing an empirical grouping method. Heredity, 38, 309-319.

LIN, C. S. AND THOMPSON, B. K. 1975. An empirical method of grouping genotypes based on a linear function of the genotype-environment interaction. Heredity, 34, 255-263.

MATHER, K. 1975. Genotype $\times$ environment interactions. II. Some genetical consideration Heredity, 35, 31-53.

MATHER, K. AND CALIGARI, P. D. S, 1976. Genotype $\times$ environment interactions. IV. The effect of the background genotype. Heredity, 36, 41-48.

MATHER, K. AND JONES, R. M. 1958. Interaction of genotype and environment in continuous variation. I. Description. Biometrics, 14, 343-359.

MATHER, K. AND JINKS, J. L. 1971. Biometrical genetics, Second edition. Chapman and Hall, London. 